\title{
Ventral-Dorsal Neural Networks: Object Detection via Selective Attention
}

\author{
Mohammad K. Ebrahimpour \\ UC Merced \\ mebrahimpour@ucmerced.edu \\ Jiayun Li \\ UCLA \\ jiayunli@ucla.edu \\ Jackson L. Reese \\ Azadeh Moghtaderi \\ Ancestry.com \\ Ancestry.com \\ jreesedancestry.com \\ amoghtaderi@ancestry.com \\ David C. Noelle \\ UC Merced \\ dnoelle@ucmerced.edu
}

\author{
Yen-Yun Yu \\ Ancestry.com \\ yyueancestry.com \\ Ming-Hsuan Yang \\ UC Merced \\ mhyang@ucmerced.edu
}

\begin{abstract}
Deep Convolutional Neural Networks (CNNs) have been repeatedly proven to perform well on image classification tasks. Object detection methods, however, are still in need of significant improvements. In this paper, we propose a new framework called Ventral-Dorsal Networks (VDNets) which is inspired by the structure of the human visual system. Roughly, the visual input signal is analyzed along two separate neural streams, one in the temporal lobe and the other in the parietal lobe. The coarse functional distinction between these streams is between object recognition - the "what" of the signal - and extracting location related information - the "where" of the signal. The ventral pathway from primary visual cortex, entering the temporal lobe, is dominated by "what" information, while the dorsal pathway, into the parietal lobe, is dominated by "where" information. Inspired by this structure, we propose the integration of a "Ventral Network" and a "Dorsal Network", which are complementary. Information about object identity can guide localization, and location information can guide attention to relevant image regions, improving object recognition. This new dual network framework sharpens the focus of object detection. Our experimental results reveal that the proposed method outperforms state-of-the-art object detection approaches on PASCAL VOC 2007 by $8 \%$ (mAP) and PASCAL VOC 2012 by 3\% (mAP). Moreover, a comparison of techniques on Yearbook images displays substantial qualitative and quantitative benefits of VDNet.
\end{abstract}

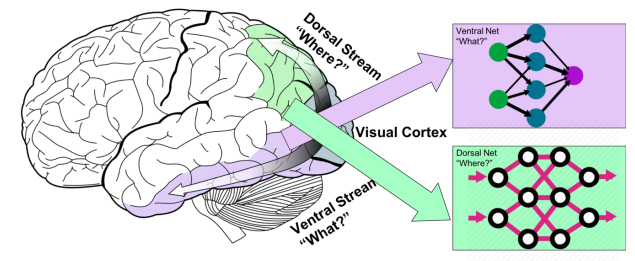

Figure 1. Primary visual cortex and two processing streams. The "ventral stream" projects into the temporal lobe, and the "dorsal stream" extends into the parietal lobe. These interacting pathways inspire the use of a "Ventral Net" and a "Dorsal Net".

\section{Introduction}

Focus matters. In order to accurately detect objects in an image, we need to know which parts of the image are important and then focus on those parts to find objects of interest. Many approaches to selective attention in artificial neural networks are computationally expensive, however, limiting their utility for online applications.

In recent years, deep Convolutional Neural Networks (CNNs) have been shown to be effective at image classification, accurately performing object recognition even with thousands of object classes when trained on a sufficiently rich dataset of labeled images [19, 29, 16, 17]. One advantage of CNNs is their ability to learn complete functional mappings from image pixels to object categories, without any need for the extraction of hand-engineered image features [28]. To facilitate learning through stochastic gradient descent, CNNs embody complex nonlinear functions which are approximately (due to activation functions like Relu) differentiable with respect to network parameters.

Image classification is only one of the core problems 
of computer vision, however. Beyond object recognition [29, 16, 17], there are applications for such capabilities as semantic segmentation [9, 31, 14], image captioning [3, 1, 32], and object detection [12, 25, 23, 24]. The last of these involves locating and classifying all of the relevant objects in an image. This is a challenging problem that has received a good deal of attention [25, 12, 13, 23]. Since there is rarely a priori information about where objects are located in an image, most approaches to object detection conduct search over image regions, seeking objects of interest with different sizes and at different scales. For example, "region proposal" frameworks, like Faster-RCNN [25], need to pass a large number of candidate image regions through a deep network in order to determine which parts of the image contain the most information concerning objects of interest. An alternative approach involves one shot detectors, like Single Shot Detectors (SSD) [21] and You Only Look Once (YOLO) [23]. These use networks to examine all parts of the image via a tiling mechanism. For example, YOLO conducts a search over potential combinations of tiles. In a sense, most off-the-shelf object detection algorithms distribute attention to all parts of the image equally, since there is no prior information concerning where objects of interest might be found.

In contrast, there is much evidence that the human visual system is equipped with a useful spatial selective attention capability that guides processing to relevant parts of the scene while effectively ignoring irrelevant parts [5]. Some theories of spatial attention focus on divergent but interacting neural pathways in the brain's visual system. Projecting from primary visual cortex in the occipital lobe, two neural streams emerge, as shown in Figure 1. One stream, extending ventrally into the temporal lobe, appears to largely encode what is in the scene, recognizing objects. The other stream, passing dorsally into the parietal lobe, approximately captures information about where objects are located. Some computational neuroscience accounts characterize spatial attention as naturally arising from interactions between these two neural pathways [22]. Inspired by this theory of attention, we propose a novel object detection framework that integrates two networks that reflect the two pathways of the human visual system. The Ventral Net uses object classification information to guide attention to relevant image regions, and the Dorsal Net uses this attentional information to accurately localize and identify objects in the scene. Together, these two components form a VentralDorsal Neural Network (VDNet).

Our object detection framework can be seen as arising from the marriage of attention based object detection $[34,33]$ and supervised object detection [23, 25]. The general approach is to use ideas from attention based object detection to quickly identify parts of the image that are irrelevant, with regard to objects of interest, using this infor- mation to guide selective attention. Focusing further supervised processing on important image regions is expected to both allow for the allocation of computational resources in a more informed manner and produce more accurate object detection by removing potentially distracting background pixels. Our selective attention process is based on Sailency Detection in activation maps, such as Class Activation Maps [37]. Guided by the resulting attentional information, we use supervised object detection to achieve high object detection performance. Thus, this framework can be seen as a combination of a top-down attention based model and the bottom-up detection based approach. As far as we know, the proposed approach is novel. Moreover, our method performs favorably against state-of-the-art object detection approaches by a large margin. In overview, we make these contributions:

- Our approach uses a top-down saliency analysis to identify irrelevant image regions, introducing a selective attention mechanism that masks out noise and unimportant background information in the image.

- Our measure of top-down saliency involves a sensitivity analysis of a trained object classification network with regard to the Gestalt Total (GT) activation produced by the network.

- Our method performs very favorably in comparison to state-of-the-art approaches, demonstrating an improvement of up to $8 \%$ (mAP) in comparsion to the best of those approaches.

The rest of this paper is organized as follows. Section 2 reviews related work. In Section 3, our proposed framework for object detection is described. Comparisons of the performance of VDNet to that of state-of-the-art methods are reported in Section 4. Final conclusions are offered in Section 5.

\section{Related Work}

State-of-the-art object detection approaches are based on deep CNNs [25, 23, 24, 14]. Leading object detection algorithms can be roughly divided into two categories: attention based object detection \& supervised object detection.

\subsection{Attention Based Object Detection}

Attention based object detection methods depend on a set of training images with associated class labels but without any annotations, such as bounding boxes, indicating the locations of objects. The lack of ground truth bounding boxes is a substantial benefit of this approach, since manually obtaining such information is costly.

One object detection approach of this kind is the Class Activation Map (CAM) method [37]. This approach is 


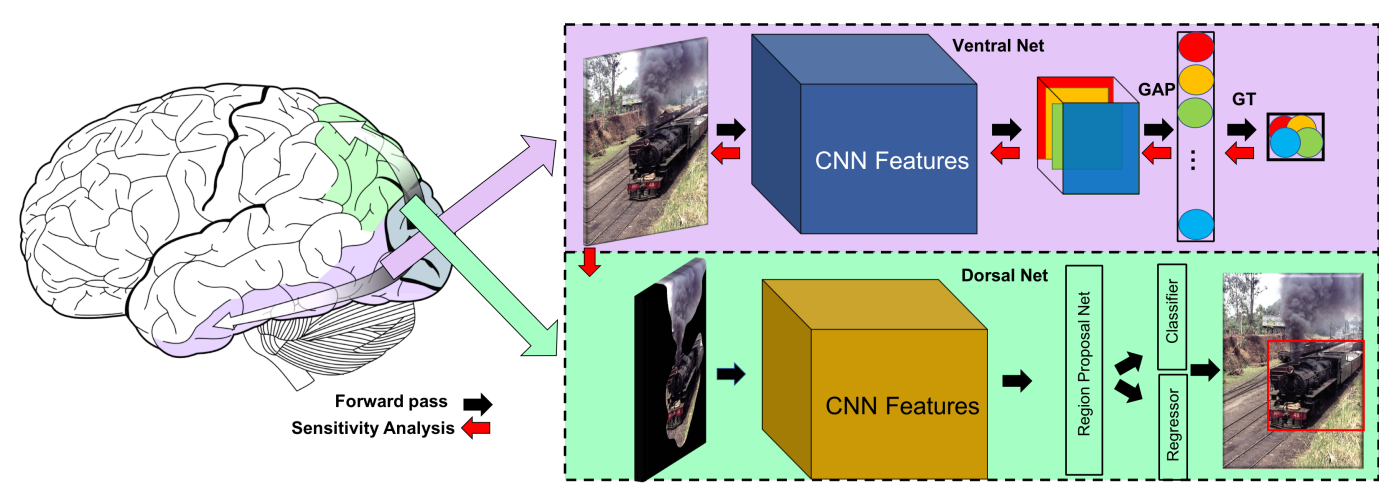

Figure 2. The Ventral-Dorsal Network (VDNet) for Object Detection. The Ventral Net filters out irrelevant parts of the image based on a sensitivity analysis of the Gestalt Total (GT) activation. The Dorsal Net then searches for objects of interest in the remaining regions.

grounded in the observation that the fully connected layers that appear near the output of typical CNNs largely discard spatial information. To compensate for this, the last convolutional layer is scaled up to the size of the original image, and Global Average Pooling (GAP) is applied to the result. A linear transformation from the resulting reduced representation to class labels is learned. The learned weights to a given class output are taken as indicating the relative importance of different filters for identifying objects of that class. For a given image, the individual filter activation patterns in the upscaled convolutional layer are entered into a weighted sum, using the linear transformation weights for a class of interest. The result of this sum is a Class Activation Map that reveals image regions associated with the target class.

The object detection success of the CAM method has been demonstrated, but it has also inspired alternative approaches. The work of Selvaraju et al. [27] suggested that Class Activation Maps could be extracted from standard image classification networks without any modifications to the network architecture and additional training to learn filter weights. The proposed Grad-CAM method computes the gradients of output labels with respect to the last convolutional layer, and these gradients are aggregated to produce the filter weights needed for CAM generation. This is an excellent example of saliency based approaches that interpret trained deep CNNs, with others also reported in the literature $[38,35,36]$.

As previously noted, attention based object detection methods benefit from their lack of dependence on bounding box annotations on training images. They also tend to be faster than supervised object detection approaches, producing results by interpreting the internal weights and activation maps of an image classification CNN. However, these methods have been found to be less accurate than supervised object detection techniques.

\subsection{Supervised Object Detection}

Supervised object detection approaches require training data that include both class labels and tight bounding box annotations for each object of interest. Explicitly training on ground truth bounding boxes tends to make these approaches more accurate than weakly supervised methods. These approaches tend to be computationally expensive, however, due to the need to search through the space of image regions, processing each region with a deep CNN. Tractability is sought by reducing the number of image regions considered, selecting from the space of all possibe regions in an informed manner. Methods vary in how the search over regions is constrained.

Some algorithms use a region proposal based framework. A deep CNN is trained to produce both a classification output and location bounding box coordinates, given an input image. Object detection is performed by considering a variety of rectangular regions in the image, making use of the CNN output when only the content in a given region is presented as input to the network. Importantly, rather than consider all possible regions, the technique depends on a $r e$ gion proposal algorithm to identify the image regions to be processed by the CNN. The region proposal method could be either an external algorithm like Selective Search [30] or it could be an internal component of the network, as done in Faster-RCNN [25]. The most efficient object detection methods of this kind are R-CNN [13], Fast-RCNN [12], Faster-RCNN [25], and Mask-RCNN [14]. Approaches in this framework tend to be quite accurate, but they face a number of challenges beyond issues of speed. For example, in an effort to propose regions containing objects of one of the known classes, it is common to base region proposals on information appearing late in the network, such as the last convolutional layer. The lack of high resolution spatial information late in the network makes it difficult to detect small objects using this approach. There are a number of research projects that aim to address this issue by combining low level features and high level ones in various ways $[38,20]$.

Rather than incorporating a region proposal mechanism, some supervised methods perform object detection in one 
feed-forward pass. A prominent method of this kind is YOLO, as well as its extensions [23, 24]. In this approach, the image is divided into tiles, and each tile is annotated with anchor boxes of various sizes, proposing relevant regions. The resulting information, along with the image tiles, are processed by a deep network in a single pass in order to find all objects of interest. While this technique is less accurate than region proposal approaches like Faster-RCNN, it is much faster, increasing its utility for online applications.

A comparison of these two general approaches to object detection displays a clear trade-off between accuracy and computational cost (speed). This gives rise to the question of whether this trade-off can be avoided, in some way. In this paper, we propose combining attention based object detection and supervised object detection in a manner that leverages the best features of both. Inspired by the human visual system and its interacting ventral and dorsal streams, we propose using a fast attention based approach to mask out irrelevant parts of the original image. The resulting selective attention greatly reduces the space of image regions to be considered by a subsequent supervised network.

\section{Method}

\subsection{Overview}

Human vision regularly employs selective attention. Without introspective awareness of the process, our brains appear to quickly focus visual processing on important parts of a scene, apparently masking out unimportant parts. Our proposed object detection framework is inspired by this phenomenon. It uses a kind of selective attention to quickly eliminate distractions, backgrounds, and irrelevant areas from the image before object detection is performed, providing a sharper focus to the process. VDNet incorporates two distinct networks: one for quickly guiding selective attention and a second for classifying and localizing objects of interest.

The Ventral Net is used to determine how to apply selective attention to the image. Ventral Net is a deep CNN that first extracts convolutional features from the input image and then aggregates the results into a Gestalt Total (GT) output. A sensitivity analysis is then performed, identifying those pixels in the original image that have the greatest immediate influence on the GT value. A simple smoothing operation translates the clouds of salient pixels into relevant regions in the image. The result is a guide for selective attention in the original image space. It provides an indication of which parts of the image are important and which are irrelevant.

The Dorsal Net performs supervised object detection, but it does so on a modified version of the image in which irrelevant regions, as determined by the Ventral Net, have been simply masked out. Selective attention speeds the region proposal process by restricting consideration to unmasked portions of the image. The result is class labels and bounding boxes for the objects of interest in the image.

In the remainder of this section, our new framework is described in detail. VDNet is illustrated in Figure 2. The following notation is used: $X \in \mathcal{R}^{m \times n \times c}$ is the image input of the system, where $m, n$, and $c$ are the width, height, and number of channels, respectively; an associated ground truth class label is $z$, and $b \in \mathcal{R}^{4}$ is the associated ground truth bounding box coordinates of the object in the input image.

\subsection{Ventral Net}

Ventral Net is responsible for guiding selective attention. It is built upon the convolutional layers of a deep CNN that has been trained to perform image classification. The last convolutional layer is used to calculate the Gestalt Total (GT) activation for the current input.

For a given image, let $f_{k}(x, y)$ denote the activation of filter $k$ in the last convolutional layer at spatial location $(x, y)$. For filter $k$, the Global Average Pooling (GAP) value is defined as:

$$
F^{k}=\sum_{x, y} f_{k}(x, y)
$$

The GT simply aggregates these values across all of the filters:

$$
G T=\sum_{k} F^{k}
$$

While this simple scalar value might seem to carry little useful information, it provides us with a simple way to identify the source pixels that have the greatest influence on the final convolutional layer activity, in a local sense. These pixels are identified through a sensitivity analysis, measuring the degree to which changes in an input pixel affect the GT. This is easily computed as follows:

$$
S=\left.\frac{\partial G T}{\partial X}\right|_{X=I_{i}}
$$

where $X$ is the network input and $I_{i} \in \mathcal{R}^{m \times n \times c}$ is the $i^{t h}$ image in the dataset. The result of this sensitivity analysis is $S \in \mathcal{R}^{m \times n \times c}$. Since we are interested in all the important pixels in the image; therefore, we work with the absolute values of $S$. Just as methods for stochastic gradient descent can involve the calculation of gradients of the output error, for a given input, with regard to network weights using a single forward pass and a single backward pass through the network, the value of $S$ can be quickly calculated for each image in the dataset.

Derivatives are calculated for all of the inputs to the network, which typically include three channels per pixel (i.e., RGB). Since the purpose of Ventral Net is to guide spatial 
attention, a single measure of relevance for each pixel location, not each channel, is needed. Thus, some way to aggregate across channels is needed. We have considered two different methods of aggregation. The first involves averaging derivative values across channels:

$$
\hat{S}_{x, y}=\frac{1}{k} \sum_{k} S_{x, y, k}
$$

where $k$ is the number of channels in the input image (i.e., 3 for RGB) and $\hat{S}$ is the $\mathcal{R}^{m \times n}$ result of aggregating derivatives. An alternative aggregation method is to use the maximum derivative across channels:

$$
\hat{S}_{x, y}=\max _{k}\left(S_{x, y, k}\right)
$$

The resulting $\hat{S}$ provides a measure of relevance at the individual pixel level. (The evaluation experiments reported in this paper all used the averaging approach to aggregation.) In order to translate this information into larger regions of relevance, we smooth $\hat{S}$ by convolving it with a Gaussian filter, resulting in $\tilde{S}$. To extract distinct regions from the resulting smoothed attention map, pixels need to be classified as relevant or irrelevant. For simplicity, this is done by setting to zero any value in $\tilde{S}$ below the mean over all pixels and setting the other values in $\tilde{S}$ to one. The resulting binary mask is duplicated across the number of channels in the original image, aligning the dimensionality of the mask and the image.

The mask is applied to the original image through simple element-wise multiplication. Formally:

$$
\hat{I}=I \odot \tilde{S}
$$

where $I$ is the original image and $\hat{I}$ is the masked image. This modified image then becomes the input to Dorsal Net.

\subsection{Dorsal Net}

Reducing the original image, $I$, to one in which irrelevant regions are masked out, $\hat{I}$, substantially reduces the space of candidate regions to consider during object detection. In Dorsal Net, the masked image is provided as input to a deep CNN trained to propose regions of interest with anchor boxes, process the contents of those regions, and output both class labels and bounding box coordinates. This is done using the methods of Faster-RCNN [25]. Dorsal Net is trained using a dataset of images that are annotated with both ground truth class labels and ground truth bounding boxes. Network parameters are selected so as to minimize a combination of the classification loss and the regression loss arising from the output of bounding box coordinates.
The complete objective function is:

$$
\begin{array}{r}
L\left(p_{i}, t_{i}\right)=\frac{1}{N_{c l s}} \sum_{i} L_{c l s}\left(p_{i}, p_{i}^{*}\right) \\
+\lambda \frac{1}{N_{\text {reg }}} \sum_{i} p_{i}^{*} L_{r e g}\left(t_{i}, t_{i}^{*}\right) .
\end{array}
$$

where $i$ is the index of an anchor box appearing in the current training mini-batch and $p_{i}$ is the predicted probability of anchor $i$ containing an object of interest. The ground truth label $p_{i}^{*}$ is 1 if anchor $i$ is positive for object presence and it is 0 otherwise. The predicted bounding box is captured by the 4 element vector $t_{i}$, and $t_{i}^{*}$ contains the coordinates of the ground truth bounding box associated with a positive anchor. The two components of the loss function are normalized by $N_{c l s}$ and $N_{\text {reg }}$, and they are weighted by a balancing parameter, $\lambda$. In our current implementation, the classification loss term is normalized by the mini-batch size (i.e., $N_{c l s}=32$ ) and the bounding box regression loss term is normalized by the number of anchor locations (i.e., $N_{\text {reg }} \approx 2,400$ ). We set $\lambda=10$, making the two loss terms roughly equally weighted.

It is worth noting that our general approach could easily support the implementation of Dorsal Net using a wide variety of alternative algorithms. The only requirement is that the object detection algorithm must be able to accept masked input images. For the results presented in this paper, we have used a leading region proposal based approach due to the high accuracy values reported for these methods in the literature. Having Ventral Net reduce the number of proposed regions is expected to speed the object detection process, and it may also improve accuracy by removing from consideration irrelevant portions of the image.

The general architecture of VDNet appears in Figure 2. Pseudocode for the proposed VDNet method is provided in Algorithm 1. An experimental evaluation of VDNet appears next.

\section{Experimental Evaluation}

\subsection{Experiment Design and Implementation}

We evaluated VDNet on PASCAL VOC 2007 [6], PASCAL VOC 2012 [7], and on a dataset of Yearbook images.

The PASCAL VOC 2007 dataset has 20 classes and 9,963 images which have been equally split into a training/validation set and a test set. The PASCAL VOC 2012 dataset contains 54,900 images from 20 different categories, and it has been split approximately equally into a training/validation set and a test set. An additional examined dataset is a private Yearbook dataset owned by Ancestry.com Operations Inc., used with permission. This dataset has more than 75 million yearbook page images. We trained a separate model to detect portraits and group photos in these images. 

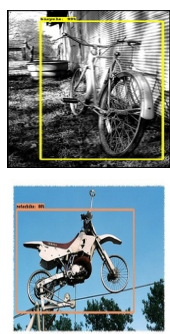
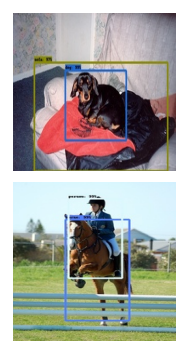
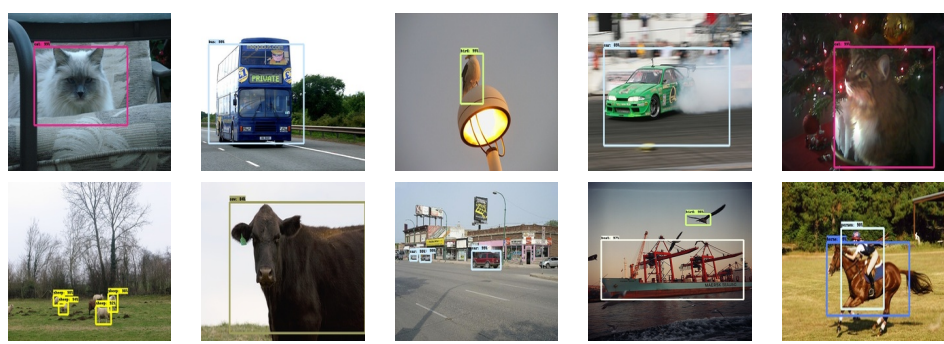

2007 Validation Images

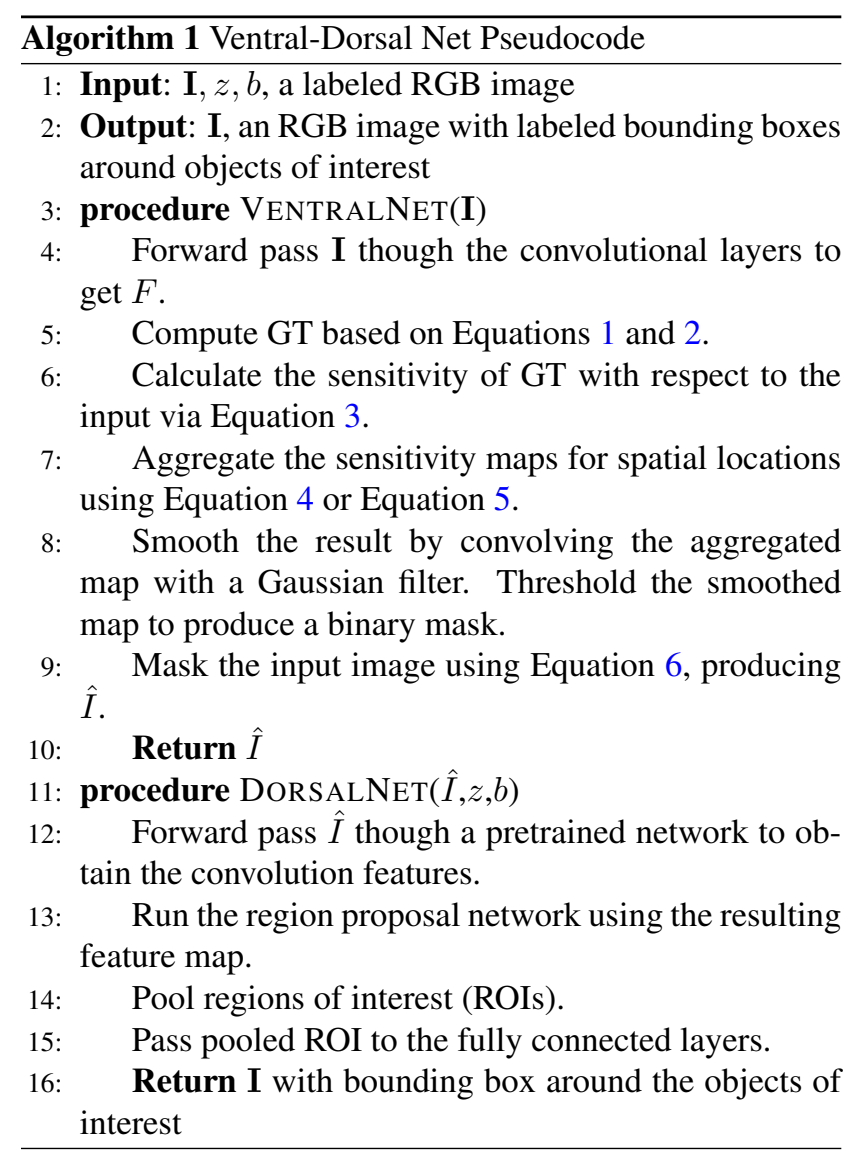

For PASCAL VOC 2007, we conducted training on the union of the VOC 2007 trainval set and the VOC 2012 trainval set, and we evaluated the result using the VOC 2007 test set. (This regimen is standard practice for these datasets.) For PASCAL VOC 2012, we performed training on its trainval set, and we evaluated the result on its test set. For the Yearbook dataset, we trained on 1 million images, and we evaluated the results on 10,000 test set images.

To evaluate performance, we used the standard mean average precision (mAP) measure. For all datasets we report $\mathrm{mAP}$ scores using IoU thresholds at 0.5.

For networks with $224 \times 224$ image inputs, using PASCAL VOC, we trained the model with a mini-batch size of 16 due to GPU memory constraints. We started the learning rate at $3 \times 10^{-4}$ for the first 900,000 epochs. We decreased it to $3 \times 10^{-5}$ until epoch $1,200,000$. Then, we decreased it to $3 \times 10^{-6}$ until epoch $2,000,000$. In all cases, we used a momentum optimizer value of 0.9 .

\subsection{PASCAL VOC 2007}

\subsubsection{Comparative Performance Results}

The results of the PASCAL VOC 2007 dataset evaluation appear in Table 1. For the Ventral Net, we utilized VGG19 pretrained on the ImageNet dataset. We removed the fully connected layers and softmax calculation from VGG19, and we calculated GT based on the last convolutional layer. The Ventral Net sensitivity analysis was performed on the resulting network. No fine tuning of parameters was done. For the Dorsal Net we used Resnet 101 pretrained on the ImageNet dataset as the backbone network for object detection. The model was trained on 8 GPUs hosted by Amazon Web Services (AWS) for about one week.

We compared our performance results with those reported for a variety of state-of-the-art approaches to object detection. Our primary baseline was Faster-RCNN using a Resnet 101 network trained on PASCAL VOC 2007. As shown in Table 1, the selective attention process of our approach (VDNet) resulted in substantially better performance in comparison to Faster-RCNN and other methods. VDNet appears to be more accurate at detecting larger objects than smaller ones, perhaps because the region proposal network based its output on the last (lowest resolution) con-
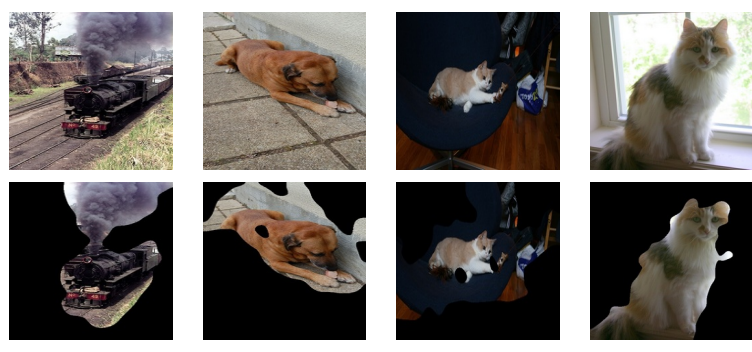

Figure 4. Some examples of selective attention image masking based on Ventral Net sensitivity analyses. The first row shows original images, and the second row displays the results of Ventral Net based masking. 


\begin{tabular}{|c|c|c|c|c|c|c|c|c|c|c|c|c|c|c|c|c|c|c|c|c|c|c|}
\hline Method & Network & mAP & areo & bike & bird & boat & bottle & bus & car & cat & chair & cow & table & $\operatorname{dog}$ & horse & mbike & person & plant & sheep & sofa & train & tv \\
\hline Faster [25] & VGG & 73.2 & 76.5 & 79 & 70.9 & 65.5 & 52.1 & 83.1 & 84.7 & 86.4 & 52 & 81.9 & 65.7 & 84.8 & 84.6 & 77.5 & 76.7 & 38.8 & 73.6 & 73.9 & $\overline{83}$ & 72.6 \\
\hline ION [2] & VGG & 75.6 & 79.2 & 83.1 & 77.6 & 65.6 & 54.9 & 85.4 & 85.1 & 87 & 54.4 & 80.6 & 73.8 & 85.3 & 82.2 & 82.2 & 74.4 & 47.1 & 75.8 & 72.7 & 84.2 & 80.4 \\
\hline Faster [15] & Residual-101 & 76.4 & 79.8 & 80.7 & 76.2 & 68.3 & 55.9 & 85.1 & 85.3 & 89.8 & 56.7 & 87.8 & 69.4 & 88.3 & 88.9 & 80.9 & 78.4 & 41.7 & 78.6 & 79.8 & 85.3 & 72 \\
\hline MR-CNN [10] & VGG & 78.2 & 80.3 & 84.1 & 78.5 & 70.8 & 68.5 & 88 & 85.9 & 87.8 & 60.3 & 85.2 & 73.7 & 87.2 & 86.5 & 85 & 76.4 & 48.5 & 76.3 & 75.5 & 85 & 81 \\
\hline R-FCN [4] & Residual-101 & 80.5 & 79.9 & 87.2 & 81.5 & 72 & 69.8 & 86.8 & 88.5 & 89.8 & 67 & 88.1 & 74.5 & 89.8 & 90.6 & 79.9 & 81.2 & 53.7 & 81.8 & 81.5 & 85.9 & 79.9 \\
\hline SSD300 [21] & VGG & 77.5 & 79.5 & 83.9 & 76 & 69.6 & 50.5 & 87 & 85.7 & 88.1 & 60.3 & 81.5 & 77 & 86.1 & 87.5 & 83.9 & 79.4 & 52.3 & 77.9 & 79.5 & 87.6 & 76.8 \\
\hline SSD512 & VGG & 79.5 & 84.8 & 85.1 & 81.5 & 73 & 57.8 & 87.8 & 88.3 & 87.4 & 63.5 & 85.4 & 73.2 & 86.2 & 86.7 & 83.9 & 82.5 & 55.6 & 81.7 & 79 & 86.6 & 80 \\
\hline DSSD3 & sidual-1 & 78.6 & 81.9 & 84.9 & 80.5 & 68.4 & 53.9 & 85.6 & 86.2 & 88.9 & 61.1 & 83.5 & 78.7 & 86.7 & 88.7 & 86.7 & 79.7 & 51.7 & 78 & 80.9 & 87.2 & 79.4 \\
\hline DSSD513 [8] & Residual-101 & 81.5 & 86.6 & 86.2 & 82.6 & 74.9 & 62.5 & 89 & 88.7 & 88.8 & 65.2 & 87 & 78.7 & 88.2 & 89 & 87.5 & 83.7 & 51.1 & 86.3 & 81.6 & 85.7 & 83.7 \\
\hline STDN & 169 & 78.1 & 81.1 & 86.9 & 76.4 & 69.2 & 52.4 & 87.7 & 84.2 & 88.3 & 60.2 & 81.3 & 77.6 & 86.6 & 9 & 87.8 & 76.8 & 51.8 & 78.4 & 81.3 & 87.5 & 77.8 \\
\hline STDN & 69 & 79.3 & 81.2 & 88.3 & 78.1 & 72.2 & 54.3 & 87.6 & 86.5 & 88.8 & 63.5 & 83.2 & 79.4 & 86.1 & 89 & 88.0 & $1 / .3$ & 52.5 & 80.3 & 80.8 & 86.3 & 82.1 \\
\hline STDN513 [38] & DenseNet-169 & 80.9 & 86.1 & 89.3 & 79.5 & 74.3 & 61.9 & 88.5 & 88.3 & 89.4 & 67.4 & 86.5 & 79.5 & 86.4 & 89.2 & 88.5 & 79.3 & 53.0 & 77.9 & 81.4 & 86.6 & 85.5 \\
\hline VDNet & Resnet-101 & 86.2 & 95.8 & 98.1 & 98.4 & 65.1 & 94.6 & 90.1 & 96.2 & 71.7 & 72.3 & 54.6 & 97.9 & 95.6 & 89.2 & 90.1 & 93.2 & 69.1 & 89.2 & 82.1 & 93.4 .6 & 74.0 \\
\hline
\end{tabular}

Table 1. PASCAL VOC 2007 Test Detection Results. Note that the minimum dimension of the input image for Faster and R-FCN is 600, and the speed is less than 10 frames per second. SSD300 indicates the input image dimension of SSD is $300 \times 300$. Large input sizes can lead to better results, but this increases running times. All models were trained on the union of the trainval set from VOC 2007 and VOC 2012 and tested on the VOC 2007 test set.

\begin{tabular}{|c|c|c|c|c|c|c|c|c|c|c|c|c|c|c|c|c|c|c|c|c|c|}
\hline Method & mAP & aero & bike & bird & boat & bottle & bus & car & cat & chair & cow & table & dog & horse & mbike & person & plant & sheep & sofa & train & tv \\
\hline 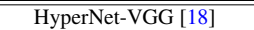 & 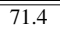 & 84.2 & 78.5 & $\overline{73.6}$ & 55.6 & "53.7 & $\overline{788.7}$ & 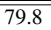 & $\overline{87.7}$ & 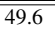 & $\overline{744.97}$ & $\begin{array}{ll}52.1 \\
\end{array}$ & 86.0 & $\begin{array}{ll}81.7 \\
\end{array}$ & 83.3 & 81.8 & 48.6 & "73.5 & 59.4 & 79.9 & 65.7 \\
\hline HyperNet-SP [18] & 71.3 & 84.1 & 78.3 & 73.3 & 55.5 & 53.6 & 78.6 & 79.6 & 87.5 & 49.5 & 74.9 & 52.1 & 85.6 & 81.6 & 83.2 & 81.6 & 48.4 & 73.2 & 59.3 & 79.7 & 65.6 \\
\hline Fast R-CNN + YOLO [23] & 70.7 & 83.4 & 78.5 & 73.5 & 55.8 & 43.4 & 79.1 & 73.1 & 89.4 & 49.4 & 75.5 & 57.0 & 87.5 & 80.9 & 81.0 & 74.7 & 41.8 & 71.5 & 68.5 & 82.1 & 67.2 \\
\hline MR-CNN-S-CNN [11] & 70.7 & 85.0 & 79.6 & 71.5 & 55.3 & 57.7 & 76.0 & 73.9 & 84.6 & 50.5 & 74.3 & 61.7 & 85.5 & 79.9 & 81.7 & 76.4 & 41.0 & 69.0 & 61.2 & 77.7 & 72.1 \\
\hline Faster R-CNN [25] & 70.4 & 84.9 & 79.8 & 74.3 & 53.9 & 49.8 & 77.5 & 75.9 & 88.5 & 45.6 & 77.1 & 55.3 & 86.9 & 81.7 & 80.9 & 79.6 & 40.1 & 72.6 & 60.9 & 81.2 & 61.5 \\
\hline NoC [26] & 68.8 & 82.8 & 79.0 & 71.6 & 52.3 & 53.7 & 74.1 & 69.0 & 84.9 & 46.9 & 74.3 & 53.1 & 85.0 & 81.3 & 79.5 & 72.2 & 38.9 & 72.4 & 59.5 & 76.7 & 68.1 \\
\hline VDNet & 73.2 & 85.1 & 82.4 & 73.6 & 57.7 & 61.2 & 79.2 & 77.1 & 85.5 & 54.9 & 79.8 & 61.4 & 87.1 & 83.6 & 81.7 & 77.9 & 45.6 & 74.1 & 64.9 & 80.3 & 73.1 \\
\hline
\end{tabular}

Table 2. PASCAL VOC 2012 Test Detection Results. Note that the performance of VDNet is about $3 \%$ better than baseline Faster-RCNN.

volutional layer. For most of the object classes, VDNet performed better than other methods by a large margin. For some classes, however, the selective attention mechanism sometimes failed to identify the relevant parts of the image, resulting in poorer performance. Some illustrative test data examples are provided in Figures 3 and 4.

\subsubsection{Discussion}

In order to further understand the contributions of the various components of VDNet, we observed the results of focal modifications to the system.

We noticed that poor performance could frequently be traced to poor selective attention masks. By varying parameters, we found that the variance of the Gaussian filter used to smooth the sensitivity analysis map played an important role. A poor choice for the variance could result in highly inappropriate attentional masks. We found that good performance could be had on the PASCAL datasets by using a variance value between 25 and 35 . It is likely, however, that this value would need to be tuned to the size and kinds of objects to be detected.

We considered performing the Ventral Net sensitivity analysis using the output class labels of the pretrained image classification network, rather than basing that analysis on the Gestalt Total (GT) activation of the last convolutional layer. It seems natural to ask for the set of pixels that contribute most to the recognition of an object of a particular class. There are a couple of reasons why we found sensitivity of GT to pixels to be a better measure. First, focusing on the last convolutional layer allowed us to produce reasonable sensitivity maps even for object classes novel to the pretrained classification network. Second, since object detection often involves scenes containing multiple objects, producing sensitivity maps based on class outputs would require the aggregation of sensitivity information for each class that might appear in the image. In the most general case, this means calculating a separate sensitivity map for each class, increasing the execution time of the Ventral Net process by a factor equal to the number of classes.

We performed a cursory examination of the role of network depth in the Dorsal Net on object detection performance on the PASCAL VOC 2007 dataset. In general, it seems as if increasing network depth increases object detection accuracy. This observation was based on comparing three different implementations of the Dorsal Net: Inception, Resnet 50, and Resnet 101. Accuracy was much better for the deeper networks, as shown in Table 3.

\begin{tabular}{c|c|c}
\hline VDNet Component & Deep Network & mAP \\
\hline Dorsal Net & Inception & 63.1 \\
Dorsal Net & ResNet50 & 71.6 \\
Dorsal Net & ResNet101 & 86.2 \\
\hline
\end{tabular}

Table 3. PASCAL VOC 2007 Test Results for Different Network Architectures

\subsection{PASCAL VOC 2012}

We also measured VDNet performance on the PASCAL VOC 2012 dataset. The Ventral Net consisted of VGG19, 
with features for calculating GT extracted from the last convolutional layer. The Dorsal Net was initialized with parameters previously learned for the PASCAL VOC 2007 evaluation, but further training was done. For the additional training, the learning rate was initialized to $3 \times 10^{-4}$ for 900,000 epochs, and then it was reduced to $3 \times 10^{-5}$ until reaching epoch $1,200,000$. The learning rate was further reduced to $3 \times 10^{-6}$ until reaching $3,000,000$ epochs. The whole training process took about 14 days on 8 GPUs hosted by AWS. This resulted in a VDNet that produced comparable or better performance that state-of-the-art methods. Performance results for PASCAL VOC 2012 are shown in Table 2.

\subsection{Yearbook Dataset}

We performed additional evaluation experiments using a dataset of grayscale Yearbook page images, testing VDNet in a somewhat different application domain. This dataset is not publicly available, but was provided by Ancestry.com Operations Inc, which possesses more than 75 million yearbook page images. Pictures on these pages appear at a variety of different angles, with different shapes, scales, and sizes. The task was to automatically detect and crop the portraits and the group photos from the pages.

Because of the differences in image properties, it did not make sense to use a network pretrained on the ImageNet dataset as the Ventral Net. Instead, we produced a classification network appropriate for this task. Our architecture included two convolutional layers, with 32 and 64 filters, respectively. The last convolutional layer was followed by max pooling and GAP, feeding a fully connected layer with Relu units followed by a softmax operation. Classification was done to recognize group photos and portraits in $224 \times 224$ image patches. A batch size of 32 was used, and training was conducted for 1,000 epochs. No regularization (e.g., weight decay, drop out) was used. The learning rate was initialized to $10^{-1}$, and it decayed exponentially every 1, 000 epochs. The Dorsal Net was Resnet 101.

We compared our VDNet to Faster-RCNN and discovered that VDNet exhibited a $7 \%$ improvement in accuracy.

\section{Conclusion}

In this paper, we highlighted the utility of incorporating fast selective attention mechanisms into object detection algorithms. We suggested that such mechanisms could potentially speed processing by guiding the search over image regions, focusing this search in an informed manner. In addition, we demonstrated that the resulting removal of distracting irrelevant material can improve object detection accuracy substantially.

Our approach was inspired by the visual system of the human brain. Theories of spatial attention that see it as arising from dual interacting "what" and "where" visual streams led us to propose a dual network architecture for object detection. Our Ventral Net consists of a pretrained image classification network, and a sensitivity analysis of this network, focusing on the Gestalt Total (GT) activation of the final convolutional layer, is performed to provide a fast identification of relevant image regions. The Ventral Net guides selective attention in the Dorsal Net, restricting the spatial regions considered by the Dorsal Net as it performs high accuracy object detection. Our approach, VDNet, integrates attention based object detection methods with supervised approaches.

The benefits of selective attention, as implemented in VDNet, are evident in performance results on the PASCAL VOC 2007 and PASCAL VOC 2012 datasets. Evaluation experiments revealed that VDNet displays greater object detection accuracy than state-of-the-art approaches, often by a large margin.

There are many opportunities for improving upon VDNet. One noteworthy weakness was observed in the detection of smaller objects, arising from the use of later, lower resolution, convolutional layers for the generation of region proposals. Ongoing research is focusing on equipping VDNet with network architectures that support learning at multiple spatial scales. The idea is to perform senstivity analyses in Ventral Net based on convolutional layers throughout the classification network, combining these in a manner that integrates information from smaller spatial scales without being distracted by more fundamental image features. It is important that the result of the sensitivity analysis continues to focus on the visual properties that are closely related to the presence of objects of interest.

Finally, it is worth noting that VDNet has a very general and flexible structure. The Ventral Net and the Dorsal Net can easily be updated to incorporate the latest advancements in image classification and object detection algorithms. In this way, we see VDNet as opening many avenues for future exploration.

\section{Acknowledgment}

This work was started as an internship research project at Ancestry.com Operations Inc. and continued at UC Merced. We would like to thank Ancestry.com Operations Inc. data science division for useful discussions and providing computational GPU resources.

\section{References}

[1] J. Aneja, A. Deshpande, and A. Schwing. Convolutional image captioning. In $C V P R, 2018$.

[2] S. Bell, C. Lawrence Zitnick, K. Bala, and R. Girshick. Inside-outside net: Detecting objects in context with skip pooling and recurrent neural networks. In CVPR, 2016. 
[3] F. Chen, R. Ji, X. Sun, Y. Wu, and J. Su. Groupcap: Groupbased image captioning with structured relevance and diversity constraints. In $C V P R, 2018$.

[4] K. J. Dai and Y. L. R-FCN. Object detection via region-based fully convolutional networks. arxiv preprint. arXiv preprint arXiv:1605.06409, 2016.

[5] R. Desimone and J. Duncan. Neural mechanisms of selective visual attention. Annual review of neuroscience, 18(1):193222, 1995.

[6] M. Everingham, L. Van Gool, C. K. I. Williams, J. Winn, and A. Zisserman. The PASCAL Visual Object Classes Challenge 2007 (VOC2007) Results. http://www.pascalnetwork.org/challenges/VOC/voc2007/workshop/index.html.

[7] M. Everingham, L. Van Gool, C. K. I. Williams, J. Winn, and A. Zisserman. The PASCAL Visual Object Classes Challenge 2012 (VOC2012) Results. http://www.pascalnetwork.org/challenges/VOC/voc2012/workshop/index.html.

[8] C.-Y. Fu, W. Liu, A. Ranga, A. Tyagi, and A. C. Berg. Dssd: Deconvolutional single shot detector. arXiv preprint arXiv:1701.06659, 2017.

[9] W. Ge, S. Yang, and Y. Yu. Multi-evidence filtering and fusion for multi-label classification, object detection and semantic segmentation based on weakly supervised learning. In $C V P R, 2018$.

[10] S. Gidaris and N. Komodakis. Object detection via a multiregion and semantic segmentation-aware cnn model. In CVPR, 2015.

[11] S. Gidaris and N. Komodakis. Object detection via a multiregion and semantic segmentation-aware cnn model. In CVPR, 2015.

[12] R. Girshick. Fast R-CNN. In $C V P R, 2015$.

[13] R. Girshick, J. Donahue, T. Darrell, and J. Malik. Rich feature hierarchies for accurate object detection and semantic segmentation. In CVPR, 2014.

[14] K. He, G. Gkioxari, P. Dollár, and R. Girshick. Mask r-cnn. In $C V P R, 2017$.

[15] K. He, X. Zhang, S. Ren, and J. Sun. Deep residual learning for image recognition. In $C V P R, 2016$.

[16] J. Hu, L. Shen, and G. Sun. Squeeze-and-excitation networks. arXiv preprint arXiv:1709.01507, 7, 2017.

[17] G. Huang, Z. Liu, L. Van Der Maaten, and K. Q. Weinberger. Densely connected convolutional networks. In CVPR, 2017.

[18] T. Kong, A. Yao, Y. Chen, and F. Sun. Hypernet: Towards accurate region proposal generation and joint object detection. In $C V P R, 2016$.

[19] A. Krizhevsky, I. Sutskever, and G. E. Hinton. ImageNet classification with deep convolutional neural networks. In NIPS, 2012.

[20] T.-Y. Lin, P. Dollár, R. B. Girshick, K. He, B. Hariharan, and S. J. Belongie. Feature pyramid networks for object detection. In CVPR, 2017.

[21] W. Liu, D. Anguelov, D. Erhan, C. Szegedy, S. Reed, C.-Y. Fu, and A. C. Berg. Ssd: Single shot multibox detector. In ECCV, 2016.

[22] R. C. O'Reilly and Y. Munakata. Computational explorations in cognitive neuroscience: Understanding the mind by simulating the brain. MIT press, 2000.
[23] J. Redmon, S. Divvala, R. Girshick, and A. Farhadi. You only look once: Unified, real-time object detection. In CVPR, 2016.

[24] J. Redmon and A. Farhadi. Yolo9000: better, faster, stronger. arXiv preprint, 2017.

[25] S. Ren, K. He, R. Girshick, and J. Sun. Faster R-CNN: Towards real-time object detection with region proposal networks. In NIPS, 2015.

[26] S. Ren, K. He, R. Girshick, X. Zhang, and J. Sun. Object detection networks on convolutional feature maps. IEEE transactions on pattern analysis and machine intelligence, 39(7):1476-1481, 2017.

[27] R. R. Selvaraju, M. Cogswell, A. Das, R. Vedantam, D. Parikh, and D. Batra. Grad-CAM: Visual explanations from deep networks via gradient-based localization. See https://arxiv.org/abs/1610.02391 v3, 7(8), 2016.

[28] P. Sermanet, D. Eigen, X. Zhang, M. Mathieu, R. Fergus, and Y. LeCun. OverFeat: Integrated recognition, localization and detection using convolutional networks. arXiv:1312.6229, 2013.

[29] K. Simonyan and A. Zisserman. Very deep convolutional networks for large-scale image recognition. CoRR, abs/1409.1556, 2014.

[30] J. R. Uijlings, K. E. Van De Sande, T. Gevers, and A. W. Smeulders. Selective search for object recognition. International journal of computer vision, 104(2):154-171, 2013.

[31] X. Wang, S. You, X. Li, and H. Ma. Weakly-supervised semantic segmentation by iteratively mining common object features. In $C V P R, 2018$.

[32] K. Xu, J. Ba, R. Kiros, K. Cho, A. Courville, R. Salakhudinov, R. Zemel, and Y. Bengio. Show, attend and tell: Neural image caption generation with visual attention. In $I C M L$, 2015.

[33] J. Yang and M.-H. Yang. Learning hierarchical image representation with sparsity, saliency and locality. In $B M V C$, 2011.

[34] J. Yang and M.-H. Yang. Top-down visual saliency via joint crf and dictionary learning. IEEE transactions on pattern analysis and machine intelligence, 39(3):576-588, 2017.

[35] X. Zhang, T. Wang, J. Qi, H. Lu, and G. Wang. Progressive attention guided recurrent network for salient object detection. In CVPR, 2018.

[36] Y. Zhang, Y. Bai, M. Ding, Y. Li, and B. Ghanem. W2f: A weakly-supervised to fully-supervised framework for object detection. In CVPR, 2018.

[37] B. Zhou, A. Khosla, A. Lapedriza, A. Oliva, and A. Torralba. Learning deep features for discriminative localization. In $C V P R, 2016$.

[38] P. Zhou, B. Ni, C. Geng, J. Hu, and Y. Xu. Scaletransferrable object detection. In CVPR, 2018. 